\title{
The Tropical Precipitation Response to Orbital Precession
}

\author{
TIMOTHY M. MERLiS \\ Princeton University, and Geophysical Fluid Dynamics Laboratory, Princeton, New Jersey \\ TAPIO SCHNEIDER AND SIMONA BORDONI \\ California Institute of Technology, Pasadena, California \\ IAN EISENMAN \\ Scripps Institution of Oceanography, University of California at San Diego, La Jolla, California
}

(Manuscript received 1 April 2012, in final form 30 July 2012)

\begin{abstract}
Orbital precession changes the seasonal distribution of insolation at a given latitude but not the annual mean. Hence, the correlation of paleoclimate proxies of annual-mean precipitation with orbital precession implies a nonlinear rectification in the precipitation response to seasonal solar forcing. It has previously been suggested that the relevant nonlinearity is that of the Clausius-Clapeyron relationship. Here it is argued that a different nonlinearity related to moisture advection by the atmospheric circulation is more important. When perihelion changes from one hemisphere's summer solstice to the other's in an idealized aquaplanet atmospheric general circulation model, annual-mean precipitation increases in the hemisphere with the brighter, warmer summer and decreases in the other hemisphere, in qualitative agreement with paleoclimate proxies that indicate such hemispherically antisymmetric climate variations. The rectification mechanism that gives rise to the precipitation changes is identified by decomposing the perturbation water vapor budget into "thermodynamic" and "dynamic" components. Thermodynamic changes (caused by changes in humidity with unchanged winds) dominate the hemispherically antisymmetric annual-mean precipitation response to precession in the absence of land-sea contrasts. The nonlinearity that enables the thermodynamic changes to affect annual-mean precipitation is a nonlinearity of moisture advection that arises because precessioninduced seasonal humidity changes correlate with the seasonal cycle in low-level convergence. This interpretation is confirmed using simulations in which the Clausius-Clapeyron relationship is explicitly linearized. The thermodynamic mechanism also operates in simulations with an idealized representation of land, although in these simulations the dynamic component of the precipitation changes is also important, adding to the thermodynamic precipitation changes in some latitudes and offsetting it in others.
\end{abstract}

\section{Introduction}

Earth's axial and apsidal precession modulates the seasonal and meridional distribution of incoming solar radiation with a $\sim 20000-\mathrm{yr}(\mathrm{kyr})$ periodicity. There is ample geological and geochemical evidence that this insolation modulation affects climate. For example, oxygen isotopes preserved in northern subtropical speleothems in Chinese caves are more depleted of heavy

Corresponding author address: Timothy M. Merlis, Princeton University and Geophysical Fluid Dynamics Laboratory, 415 Jadwin Hall, Princeton, NJ 08544.

E-mail: tmerlis@princeton.edu oxygen isotopes when perihelion occurs near the northern summer solstice (Wang et al. 2008). This has been interpreted as indicating enhanced precipitation at those times compared with the present orbital configuration, in which perihelion occurs near the southern summer solstice. Similarly, southern subtropical speleothems in Brazilian caves are more depleted of heavy oxygen isotopes when perihelion occurs near the southern summer solstice (Cruz et al. 2005; Wang et al. 2006, 2007). Thus, speleothem records suggest that precession leads to hemispherically antisymmetric precipitation changes: precipitation is enhanced in the hemisphere with summer solstice near perihelion and is reduced in the other hemisphere. However, the source of the 
oxygen isotope variations in speleothems and their connection to precipitation is unclear. A simple estimate of precipitation changes associated with precession, based on correlations over the seasonal cycle in the present climate, suggests unphysically large (>50\%) precipitation changes (Dayem et al. 2010). Simulations with isotope-enabled general circulation models (GCMs) point to the importance of complex nonlocal influences on the isotopic composition of precipitation: moisture source regions change when the climate is perturbed, which can have a greater effect on the isotopic composition of precipitation than on its amount (Legrande and Schmidt 2009; Pausata et al. 2011).

Beyond speleothems, a range of other evidence also suggests that the tropical hydrological cycle is sensitive to the seasonal redistribution of insolation associated with precession. For example, atmospheric methane concentrations preserved in ice cores vary on precessional time scales, and these variations are thought to be related to the influence of the Northern Hemisphere tropical hydrological cycle on methane emissions (e.g., Schmidt et al. 2004, and references therein). Earlier in the Holocene, when perihelion occurred during northern summer, the African Sahara was substantially wetter and had lakes (e.g., deMenocal et al. 2000; Street and Grove 1979), and subtropical Asian lakes also were deeper at that time (Gasse et al. 1991; Herzschuh 2006).

To the extent that these paleoclimate proxy records indicate annual-mean changes in precipitation or in net precipitation (precipitation minus evaporation), as is the case for lake levels, it is unclear how precession causes them. The annual-mean insolation at a given latitude does not change with precession: the factors of the squared Earth-sun distance $r^{2}$ in the conservation of angular momentum (Kepler's second law: Earth moves faster through its orbit when it is closer to the sun) and of $r^{-2}$ in the radiative energy flux reaching Earth cancel each other exactly. Therefore, if seasonal precipitation changes depended linearly on insolation changes, orbital precession would not affect annualmean precipitation. Any annual-mean precipitation change must arise through nonlinear rectification of seasonal changes. This is true even when the climate in each season individually responds linearly to insolation forcing (Jackson and Broccoli 2003): if the sensitivity to insolation forcing varies during the course of the year, the departure from a linear sensitivity with constant coefficient can give rise to a nonlinearly rectified annualmean precession response. Note also that the processes by which paleoclimate proxies are recorded can vary seasonally, which can produce precession signals in the proxies that are unrelated to annual-mean climate changes (Huybers and Wunsch 2003). For example, the speleothem oxygen isotope record may be more strongly influenced by precipitation in particular seasons or have nonlocal influences, as described above, causing it to reflect something other than annual-mean precipitation.

A conventional interpretation (COHMAP members 1988; Ruddiman 2008) of the precession signal in tropical records is that large-scale seasonal sea-breeze circulations, such as monsoons, strengthen because landsea surface temperature contrasts are enhanced when perihelion occurs in summer, leading to more intense precipitation (Fig. 1, top). However, it is unclear how precession-induced changes in a cross-equatorial seabreeze circulation would affect precipitation over oceans in the hemisphere with the dimmer summer: either the precipitation decouples from the circulation mass flux (as suggested in Fig. 1), or precipitation increases associated with an increased mass flux are regional and are offset by opposing changes elsewhere. Precipitation cannot increase in both hemispheres in the zonal mean, as this would lead to an energetically inconsistent annualmean increase in precipitation. As an alternative interpretation, it has also been suggested that strengthening (convergent) summer sea-breeze circulations affect precipitation more than strengthening (divergent) winter sea-breeze circulations because the Clausius-Clapeyron nonlinearity in the saturation specific humidity leads to larger precession-induced specific humidity changes in summer (Kutzbach and Guetter 1986).

Here we wish to constrain the magnitude of possible changes in annual-mean tropical precipitation in response to precession and identify the mechanisms responsible for them. We use an idealized atmospheric GCM and analyze the changes that occur in the statistically steady states of simulations in which the top-ofatmosphere insolation is varied to represent different precessional states representative of those that occurred in Earth's history. Our GCM is idealized, in contrast to the more comprehensive GCMs used in previous modeling studies (e.g., Kutzbach 1981; Kutzbach and Guetter 1986; Joussaume et al. 1999; Braconnot et al. 2007; Legrande and Schmidt 2009). This allows us to clearly identify the mechanisms involved in the tropical precipitation response to precession.

To understand the annual-mean precipitation response mechanistically, we follow a similar approach to that taken by Clement et al. (2004) and Hsu et al. (2010), who decomposed changes in the water vapor budget for solstice seasons into different components to determine the extent to which net precipitation changes are thermodynamic (i.e., dominated by changes in water vapor concentration with unchanged winds) or dynamic 


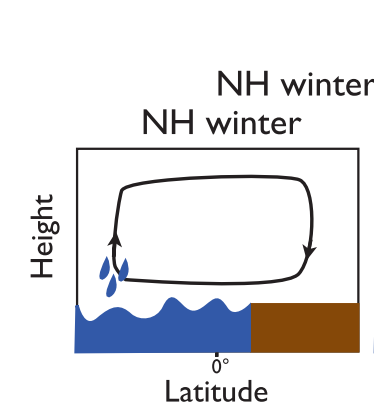

\section{Sea breeze paradigm}

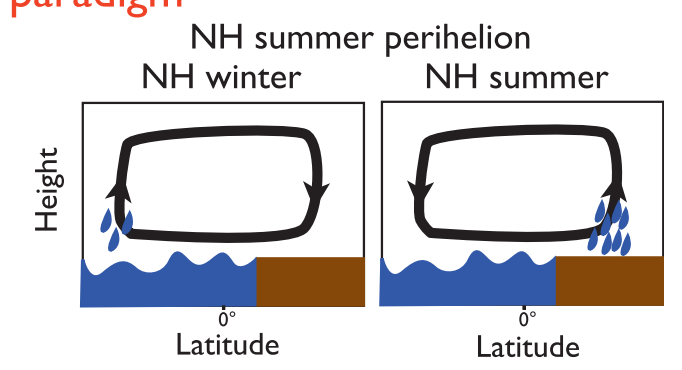

$\mathrm{NH}$ winter perihelion
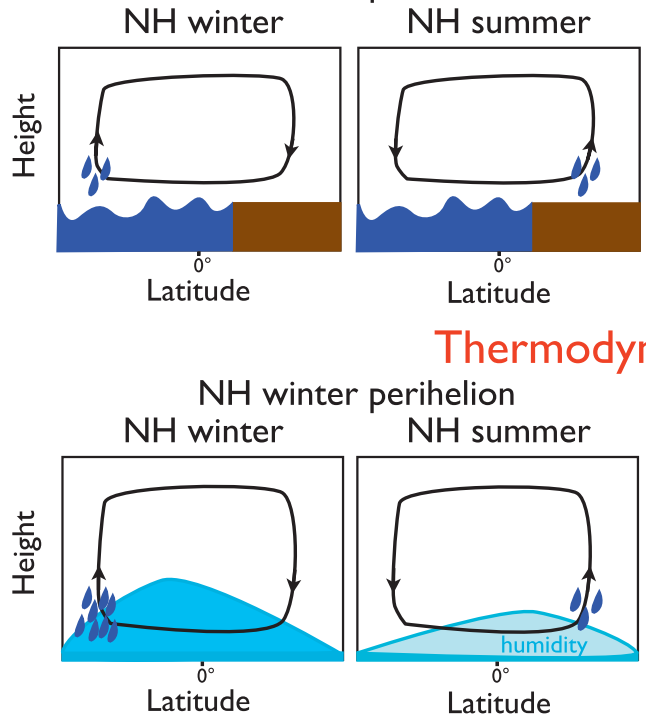

Thermodynamic mechanism

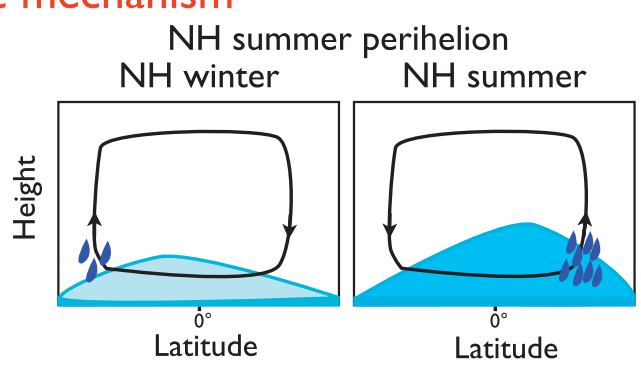

FIG. 1. Schematics of (top) the conventional seasonal sea-breeze paradigm and (bottom) the thermodynamic mechanism presented here for precession-forced tropical precipitation changes. Blue raindrops represent precipitation, with more raindrops indicating more precipitation. Black contours represent the mean meridional circulation, with thicker contours indicating stronger mass transport. In the upper panels, the brown and blue regions near the bottom represent land and ocean. In the lower panels, the blue shading represents atmospheric humidity, with darker shades indicating greater humidity.

(i.e., dominated by changes in winds with unchanged water vapor concentration). Our simulation results show that, in the absence of land-sea contrasts, thermodynamic changes dominate and give rise to annual-mean precipitation changes because precession-induced seasonal humidity changes correlate with the seasonal cycle in low-level convergence. This correlation represents an advective nonlinearity (it results because moisture advection is nonlinear), and we show that this nonlinearity rectifies seasonal insolation changes to produce annualmean precipitation changes. This provides a simple mechanism for the hemispherically antisymmetric precipitation variations in proxy records: When perihelion occurs close to summer solstice in one hemisphere, the atmosphere is warmer and moister during summer in that hemisphere (Fig. 1, bottom). Conversely, in the opposite hemisphere, annual-mean precipitation is reduced because the atmosphere is cooler and drier in its summer, when low-level moisture fluxes converge. Even with unchanged winds, this mechanism affects annualmean precipitation because the humidity changes are in phase with the seasonal cycle of the low-level convergence and ascending air masses, which lead to precipitation. We also discuss the dynamic component of precession-forced precipitation changes, which can act to augment or offset the thermodynamic changes, particularly in the presence of land-sea contrasts.

\section{Idealized GCM}

We use an idealized moist atmospheric GCM based on the Geophysical Fluid Dynamics Laboratory's (GFDL) Flexible Modeling System (FMS) spectral dynamical core (with T42 horizontal resolution and 30 levels in the GCM's $\sigma=p / p_{s}$ vertical coordinate) and the radiation scheme of GFDL's AM2 comprehensive atmospheric GCM (Anderson et al. 2004). See Merlis et al. (2013a) and Merlis et al. (2013b) for a complete description of the model. The GCM has an idealized convection scheme (Frierson 2007; O'Gorman and Schneider 2008) and a prescribed ocean heat flux convergence that is similar to observational estimates (Bordoni 2007; Bordoni and Schneider 2008). As the treatment of radiative transfer is the same as that in a comprehensive GCM, our GCM permits the water vapor feedback, but we suppress poorly constrained cloud feedbacks by prescribing timeindependent clouds (Merlis et al. 2013a).

We present results from "aquaplanet" simulations with a uniform surface with an albedo of 0.1 and with a heat capacity equivalent to $5 \mathrm{~m}$ of water. Our focus is on simulations with low surface thermal inertia because they permit convergence zones to migrate poleward seasonally, like in the Asian monsoon (Bordoni and Schneider 2008). In addition, it is useful to have somewhat exaggerated seasonal variations to clearly isolate 

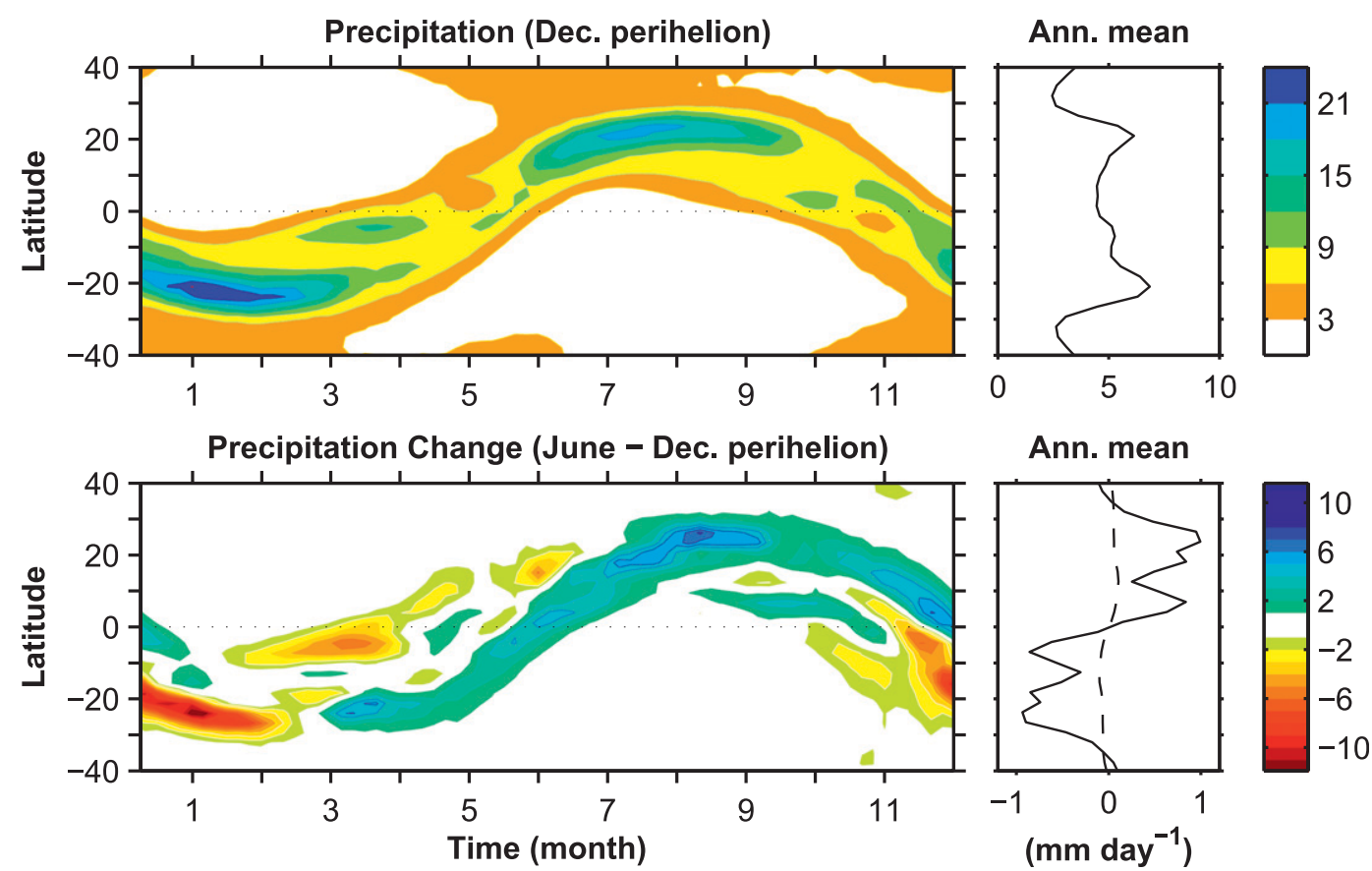

FIG. 2. (top left) Seasonal cycle of precipitation in reference simulation with perihelion in December (contour interval of $3 \mathrm{~mm} \mathrm{day}^{-1}$ ). (top right) Annual-mean precipitation in reference simulation. (bottom left) Seasonal cycle of precipitation difference between simulations with perihelion in June and December (contour interval of $1 \mathrm{~mm}$ day $^{-1}$ ). (bottom right) Annual-mean difference in precipitation (solid) and evaporation (dashed) between simulations with perihelion in June and December.

mechanisms. As a thermal inertia this small is intended to be illustrative and to compensate for some processes that are ignored (e.g., orographic influences on the circulation energetics; Privé and Plumb 2007b; Boos and Kuang 2010), we also present the results from simulations with a surface heat capacity of $20 \mathrm{~m}$ of water everywhere except for the Northern Hemisphere subtropics (from $10^{\circ}$ to $30^{\circ} \mathrm{N}$ ), where it is 100 times smaller (Merlis et al. 2013b). This is intended to represent, in a limited way, the effect of land-sea contrasts in surface properties. As the GCM boundary conditions and forcing are zonally symmetric, we analyze the zonal mean precipitation, recognizing that regional variations will be superimposed on these changes in more realistic climates that are not statistically zonally symmetric.

We vary the orbital configuration using a 360-day year. The reference orbital parameters that we use are a nearcontemporary obliquity of $23^{\circ}$, a relatively large eccentricity of 0.05 , and perihelion occurring at southern summer solstice, which is close to the contemporary value. We refer to this as December perihelion for brevity. The perturbation simulations we conduct have perihelion occurring at northern summer solstice, referred to as June perihelion. With eccentricity of 0.05 , the seasonal insolation change between December and June perihelion is $\sim 50 \mathrm{~W} \mathrm{~m}^{-2}$ in the tropics (see Fig. 2 of Merlis et al. 2013a).

\section{Precipitation changes}

\section{a. Simulation results}

Figure 2 (top) shows the seasonal cycle of precipitation in low latitudes in the reference aquaplanet simulation with December perihelion. The precipitation maximum moves seasonally into the summer hemisphere, reaching $15^{\circ}-25^{\circ}$, as in the Asian monsoon region. Because the thermal inertia of the surface is low, the convergence zone rapidly crosses the equator after the equinoxes.

When perihelion changes from December to June, the precipitation changes are phased similarly to the insolation and surface temperature changes: there is more precipitation in the warm northern summer and less precipitation in the cool southern summer (Fig. 2, bottom). The annual-mean precipitation changes by $\sim 1 \mathrm{~mm}$ day $^{-1}$ $(\sim 15 \%)$, with more annual-mean precipitation in the hemisphere in which perihelion coincides with summer solstice (Fig. 2, bottom right). In what follows, the columnintegrated water vapor budget is analyzed to determine the relevant climate nonlinearity that leads to the annual-mean precipitation changes.

In contrast to the precipitation, there is no substantial $(\leq 2 \%)$ annual-mean change in evaporation (dashed line in bottom right panel of Fig. 2). This is consistent with the absorption of surface shortwave radiation $S_{\text {surf }}$ 
being the dominant heating term and evaporation $E$ being the dominant cooling term in the surface energy balance, as in Earth's tropics. So changes in shortwave radiation are balanced by changes in evaporation: $\delta E \sim \delta S_{\text {surf }}$. Because there is no annual-mean change in shortwave radiative fluxes, time-dependent changes in evaporation-for example, greater evaporation rates in the summer with greater insolation-largely average out in the annual mean. Therefore, precipitation $P$ and net precipitation $P-E$ are essentially interchangeable in our discussion of annual means.

\section{b. Thermodynamic/dynamic decomposition of water vapor budget}

In the annual mean (denoted [.]), the net precipitation $[P-E]$ is balanced by the mass-weighted vertical integral (denoted $\{\cdot\}$ ) of the convergence of the water vapor flux $-\nabla \cdot[\mathbf{u} q]$ :

$$
[P-E]=-\int_{0}^{p_{s}} \nabla \cdot[\mathbf{u} q] \frac{d p}{g}=-\nabla \cdot\{[\mathbf{u} q]\},
$$

with horizontal wind vector $\mathbf{u}$ and specific humidity $q$. As the annual-mean changes in evaporation are small, the changes in the water vapor convergence are balanced by precipitation changes,

$$
\delta[P] \approx-\delta \nabla \cdot\{[\mathbf{u} q]\} .
$$

The Reynolds-averaged form of the water vapor flux, with $\overline{(\cdot)}$ defined as a monthly mean and $(\cdot)^{\prime}$ indicating a deviation thereof, is $\overline{\mathbf{u} q}=\overline{\mathbf{u}} \bar{q}+\overline{\mathbf{u}^{\prime} q^{\prime}}$. The perturbation water vapor flux is $\delta \overline{\mathbf{u} q}=\delta \overline{\mathbf{u}} \bar{q}+\overline{\mathbf{u}} \delta \bar{q}+\delta \overline{\mathbf{u}} \delta \bar{q}+\delta \overline{\mathbf{u}^{\prime} q^{\prime}}$, where variables not immediately preceded by $\delta$ are unperturbed, that is, control climate variables. One can further decompose the changes in specific humidity $\delta q$ into those due to relative humidity changes $\delta \mathcal{H}$ or saturation specific humidity changes $\delta q_{s}: \delta q \approx \mathcal{H} \delta q_{s}+$ $\delta \mathcal{H} q_{s}$, where the product of the perturbed quantities has been neglected, and there is a small approximation in setting the ratio of the specific humidity and the saturation specific humidity equal to the relative humidity. Combining these terms, the perturbation water vapor flux is

$$
\begin{aligned}
\delta \overline{\mathbf{u} q}= & \delta \overline{\mathbf{u}} \bar{q} \\
& +\overline{\mathbf{u}} \mathcal{H} \delta \bar{q}_{s} \\
& +\overline{\mathbf{u}} \delta \mathcal{H} \bar{q}_{s} \\
& +\delta \overline{\mathbf{u}} \delta \bar{q} \\
& +\delta \overline{\mathbf{u}^{\prime} q^{\prime}}
\end{aligned}
$$

where the right-hand side of (3) is the "dynamic" component of the changes and (4) is the "thermodynamic" component of the changes. Other changes, such as those associated with changes in relative humidity (5) or transient eddies (7), are possible, but they are relatively small in our simulations, so we do not focus on them here. This type of analysis has been performed for GCM simulations of climate changes forced by greenhouse gases (Held and Soden 2006) and by orbital precession (Clement et al. 2004).

While discussion of the precipitation response to orbital precession is often focused on strengthening monsoonal circulations, the expectation for climate warming caused by increased greenhouse gas concentrations is different. Under global warming, net precipitation in convergence zones will increase from the thermodynamic changes even if atmospheric circulations weaken, provided they weaken at a slower rate than the humidity increases (close to that of the Clausius-Clapeyron relationship). It is often the case that circulations weaken more slowly than the rate given by the Clausius-Clapeyron relationship in simulations of global warming, so it is not unusual to have increased precipitation in convergence zones and reduced mean circulations (e.g., Held and Soden 2006; O'Gorman and Schneider 2008; Seager et al. 2010; Schneider et al. 2010).

\section{c. Water vapor budget decomposition: Aquaplanet simulation results}

Figure $3 \mathrm{a}$ shows the change in the annual mean, vertically integrated water vapor convergence (equal to $[P-E])$ between the aquaplanet simulations with perihelion in June and December. As perihelion changes from December to June, the water vapor convergence increases in the Northern Hemisphere (more net precipitation) and decreases in the Southern Hemisphere (less net precipitation). The total changes are dominated by the thermodynamic component (4) of the changes (Fig. 3). This is consistent with water vapor concentrations that increase because the near-surface temperature increases, while relative humidity changes are weak, when insolation is strengthened ${ }^{1}$ (Fig. 3 of Merlis et al. 2013a). The dynamic changes (3) are smaller and of opposite sign as the thermodynamic changes (Fig. 3).

\footnotetext{
${ }^{1}$ Note that GCM simulations with fixed sea surface temperature (SST), such as those in Kutzbach (1981) and Kutzbach and Guetter (1986), cannot capture this basic relationship between insolation, temperature, and humidity. The results of comprehensive coupled GCM simulations of the mid-Holocene confirm that this aspect of fixed SST simulations is problematic: in coupled GCM simulations, precession-forced changes in surface shortwave radiation are largely balanced by changes in evaporation, which increase the near-surface atmospheric humidity (Hewitt and Mitchell 1998; Zhao et al. 2005).
} 

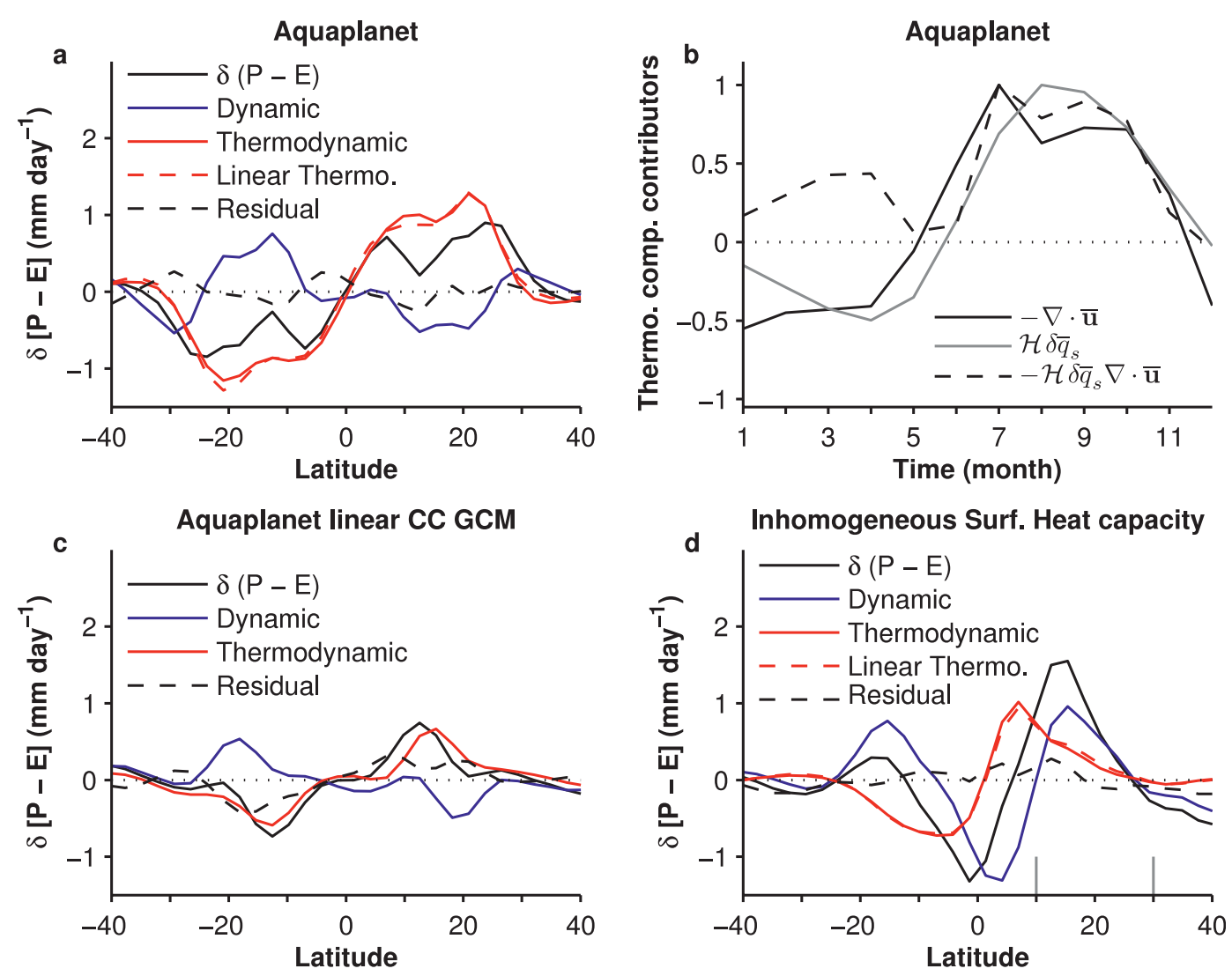

FIG. 3. (a) Decomposition of annual-mean difference in water vapor flux convergence between aquaplanet simulations with perihelion in June and December. See (3)-(7) and associated text for definitions. (b) Time series of contributors to the thermodynamic component: the convergence of mean wind $(-\nabla \cdot \overline{\mathbf{u}}$, black solid line) in the December perihelion aquaplanet simulation, the precession-induced thermodynamic change in specific humidity $\left(\mathcal{H} \delta \bar{q}_{s}\right.$, gray solid line), and the product of the two $\left(-\mathcal{H} \delta \bar{q}_{s} \nabla \cdot \overline{\mathbf{u}}\right.$, black dashed line), all averaged over the lower troposphere (from $\sigma=1$ to $\sigma=0.73$ ) and from $5^{\circ}$ to $20^{\circ} \mathrm{N}$. The time series have been divided by the maximum monthly mean values of $2.1 \times 10^{-6} \mathrm{~s}^{-1}, 5.0 \times 10^{-3}$, and $6.1 \times 10^{-9} \mathrm{~s}^{-1}$, respectively. (c) Decomposition of annualmean difference in water vapor flux convergence between aquaplanet linearized Clausius-Clapeyron GCM simulations with perihelion in June and December. (d) Decomposition of annual-mean difference in water vapor flux convergence between subtropical continent simulations with perihelion in June and December. Continent boundaries are indicated by vertical gray lines.

This is a consequence of the counterintuitive result that the simulation with the bright Northern Hemisphere summer has a weaker monsoonal circulation (Merlis et al. 2013a). The terms associated with changes in relative humidity (5), the product of perturbed mean fields (6), and transient eddies (7) are negligible (their sum is labeled "Residual" in Fig. 3).

That the thermodynamic component is large and is associated with changes in water vapor concentration might lead to the expectation that the nonlinearity that gives rise to the annual-mean changes is that of the Clausius-Clapeyron relationship, as suggested previously (Kutzbach and Guetter 1986; Prell and Kutzbach 1987). To evaluate the importance of this nonlinearity, we form a linearized thermodynamic component,

$$
\left[\overline{\mathbf{u}} \mathcal{H} \delta \bar{q}_{S}\right] \approx\left[\overline{\mathbf{u}} \mathcal{H} \bar{q}_{S} \alpha \delta \bar{T}\right],
$$

in which the saturation specific humidity $\bar{q}_{s}$ is taken as that in the control climate and a constant fractional rate of change of the saturation specific humidity with respect to temperature, $\alpha=6.5 \% \mathrm{~K}^{-1}$, is used. This value of $\alpha$ is the fractional rate of change of the saturation specific humidity at $T=290 \mathrm{~K}$ and $p=10^{5} \mathrm{~Pa}$, which are representative values for the near-surface tropical atmosphere, where most of the water vapor resides. The linearized approximation (8), in which the perturbation saturation specific humidity is a linear function of the perturbation temperature, is approximately equal to the full thermodynamic component (red dashed and solid lines in Fig. 3). Thus, it is not the case that the 
nonlinearity associated with the Clausius-Clapeyron relationship (i.e., the nonlinear dependence of the saturation specific humidity on temperature) is important. Kutzbach and Guetter (1986) and Prell and Kutzbach (1987) argued that this nonlinearity is relevant for determining the response of precipitation to orbital precession; however, for this nonlinearity to be qualitatively important, either 1) the amplitude of the seasonal cycle of tropical, near-surface water vapor needs to be substantially larger than that in Earth's present climate or 2) the precession-induced near-surface temperature changes need to be larger than the range of results previously published, which are typically less than $\sim 3 \mathrm{~K}$ (Braconnot et al. 2007; Legrande and Schmidt 2009).

To demonstrate what the relevant nonlinearity is that gives rise to the annual-mean precipitation changes, we further decompose the thermodynamic component of the water vapor flux convergence into the advection of the perturbation specific humidity $-\overline{\mathbf{u}} \cdot \nabla\left(\mathcal{H} \delta \bar{q}_{s}\right)$ and the product of the perturbation specific humidity and the convergence of the mean wind $-\left(\mathcal{H} \delta \bar{q}_{s}\right) \nabla \cdot \overline{\mathbf{u}}$ :

$$
-\nabla \cdot\left(\overline{\mathbf{u}} \mathcal{H} \delta \bar{q}_{s}\right)=-\overline{\mathbf{u}} \cdot \nabla\left(\mathcal{H} \delta \bar{q}_{s}\right)-\left(\mathcal{H} \delta \bar{q}_{s}\right) \nabla \cdot \overline{\mathbf{u}} .
$$

In the simulations, the vertically integrated convergence term $-\left\{\left(\mathcal{H} \delta \bar{q}_{s}\right) \boldsymbol{\nabla} \cdot \overline{\mathbf{u}}\right\}$ dominates the vertically integrated advection term $-\left\{\overline{\mathbf{u}} \cdot \nabla\left(\mathcal{H} \delta \bar{q}_{s}\right)\right\}$ by a factor of $\sim 5$.

Figure $3 \mathrm{~b}$ shows the time series of the convergence and of the precession-induced thermodynamic change in specific humidity in the tropical lower troposphere in the Northern Hemisphere. Both quantities are now averaged over the lower troposphere because the scale height of water vapor is small and because continuity relates the low-level convergence to vertical motion. Their product largely determines the dominant thermodynamic component of the perturbation water vapor budget (Fig. 3a). The two time series are in phase and are positively correlated. Thus, the nonlinearity that gives rise to the annual-mean response of precipitation is the advective nonlinearity of the product of the unperturbed, seasonally varying mean meridional circulation and the seasonally varying saturation specific humidity perturbation. The nonlinearity is, equivalently, caused by the correlation of the seasonal cycle of climatological ascent and the precession-induced perturbation specific humidity (Fig. 1, bottom).

Both the time series of the mean wind convergence and of the precession-induced thermodynamic change in specific humidity are asymmetric about zero, with the magnitude of the most positive values exceeding the magnitude of the most negative values (Fig. 3b). The asymmetry in the convergence of the mean meridional wind arises because the ascending branch of the Hadley cell has a smaller meridional scale than the descending branch (Fig. 5 of Merlis et al. 2013a). The asymmetry in the perturbation specific humidity arises because the control climate's lower-tropospheric relative humidity $\mathcal{H}$ is greater in summer than in winter and because the saturation specific humidity depends nonlinearly on temperature, although this nonlinearity is not quantitatively important for the annual-mean precipitation changes (Fig. 3a). The asymmetries in the low-level convergence and specific humidity time series modify the magnitude of the annual-mean precipitation changes, but they are not what is responsible for the fact that there are nonzero annual-mean changes: there would still be annual-mean precipitation changes if the two time series were sinusoids symmetric about zero, provided the phase relationship remained the same. For example, when the saturation specific humidity's nonlinear temperature dependence is explicitly removed, the thermodynamic component of the annual-mean precipitation changes little (Fig. 3a).

The thermodynamic changes dominate the precessioninduced changes in annual-mean water vapor convergence in the aquaplanet simulations, though one might have expected that the nonlinear dynamics of the crossequatorial or monsoonal Hadley circulation (Lindzen and Hou 1988; Bordoni and Schneider 2008) are an important factor in the climate response to orbital precession. The Hadley circulation changes and the mechanisms determining them in the aquaplanet simulations are examined in detail in Merlis et al. (2013a), and we briefly summarize the key results here. Interestingly, the circulations defy the expectation that stronger meridional insolation gradients and surface temperature gradients lead to stronger circulations: the cross-equatorial circulations are weaker in the summer with perihelion. The monsoonal circulation is near the angular momentumconserving limit, so it responds to changes in the top-ofatmosphere energy budget through its energy transport (Held and Hou 1980; Schneider 2006). However, the ratio of the circulation's energy transport to the mass transport (the gross moist stability) is also free to change, so knowledge of the changes in the energy transport does not directly constrain the changes in the mass transport (Held 2000). The gross moist stability in the bright summer is larger than that in the dim summer (i.e., the circulation in the bright summer transports energy more efficiently) by an amount that is more than sufficient to balance the change in energy transport that is required by the top-of-atmosphere radiation perturbation from precession; the circulation mass transport in the bright summer can therefore be weaker than that of the dim summer (Merlis et al. 2013a). The gross moist stability depends, in part, on the near-surface gradients 
of temperature and humidity (Held 2001). The stronger near-surface gradients in these quantities in the bright summer lead to a larger gross moist stability (Merlis et al. 2013a). While we have a physically based mechanism to explain the surprising circulation changes found in the aquaplanet GCM simulations, it is natural to wonder if these are an artifact of using an idealized GCM. A similar result (weaker cross-equatorial Hadley circulation in a precession-forced bright summer) has been found in comprehensive GCM simulations (Clement et al. 2004; Brayshaw et al. 2010), though this may not be a robust GCM response (Ashkenazy et al. 2010; Khon et al. 2010).

\section{d. Aquaplanet simulations with linearized Clausius-Clapeyron relationship}

The results of the previous section illustrate that the nonlinearity of the Clausius-Clapeyron relationship is not essential to the tropical precipitation response to orbital precession: when the changes in the water vapor budget are diagnosed by combining a linear saturation specific humidity with the GCM-simulated temperature change, the results are similar to those obtained using the GCM-simulated specific humidity, which includes the Clausius-Clapeyron nonlinearity. In this section, we present the results of aquaplanet simulations that use a version of the GCM with an explicitly linearized Clausius-Clapeyron relationship to provide additional support for the conclusion that the nonlinearity of the Clausius-Clapeyron relationship is not essential to the tropical precipitation changes forced by orbital precession. A GCM with a linearized Clausius-Clapeyron relationship will simulate a mean climate that is significantly different from a corresponding simulation with a nonlinear Clausius-Clapeyron relationship because the temperature range of the atmosphere is substantial $(\sim 100 \mathrm{~K})$; however, the response to climate perturbations may be qualitatively similar if the temperature perturbations are small $(\$ 5 \mathrm{~K})$, so the Clausius-Clapeyron nonlinearity may not be important in determining the perturbation response. We are not aware of any previously published GCM experiments that use a linear Clausius-Clapeyron relationship and believe this may be a conceptually valuable approach for mechanistic analysis of other climate change simulations (e.g., greenhouse gas changes).

The saturation vapor pressure formula of the standard version of the idealized GCM is

$$
e_{s}(T)=e_{0} \exp \left[-\frac{L}{R_{v}}\left(\frac{1}{T}-\frac{1}{T_{0}}\right)\right],
$$

with $e_{0}=610.78 \mathrm{~Pa}, L=2.5 \times 10^{6} \mathrm{~J} \mathrm{~kg}^{-1}, R_{v}=$ $461.50 \mathrm{~J} \mathrm{~K}^{-1} \mathrm{~kg}^{-1}, T_{0}=273.16 \mathrm{~K}$, and standard variable definitions. In the linearized Clausius-Clapeyron simulations presented in this section, the saturation vapor pressure formula is linearized for temperatures greater than $T_{0}$ :

$$
e_{s}^{\operatorname{lin}}(T)=\left\{\begin{array}{lll}
e_{0} \exp \left[-\frac{L}{R_{v}}\left(\frac{1}{T}-\frac{1}{T_{0}}\right)\right] & \text { if } & T<T_{0} \\
e_{0}+\left.\partial_{T} e_{s}\right|_{T=T_{0}} \times\left(T-T_{0}\right) & \text { if } & T>T_{0},
\end{array}\right.
$$

so that the saturation vapor pressure is linear in temperature for temperatures above $T_{0}$, and the first derivative of the vapor pressure is continuous for all temperatures. The temperature range over which the saturation vapor pressure is linear is relevant for tropical precipitation because most of it occurs at temperatures greater than $T_{0}$.

Figure $3 \mathrm{c}$ shows that the response of net precipitation to orbital precession in the simulations with a linearized Clausius-Clapeyron relationship is similar to the simulations with a nonlinear Clausius-Clapeyron relationship (Fig. 3a): the tropical precipitation response to orbital precession is antisymmetric about the equator, and the thermodynamic component dominates the increase in net precipitation $\left(\sim 0.5 \mathrm{~mm} \mathrm{day}^{-1}\right)$ in the hemisphere with the bright summer. The dynamic component offsets part of the thermodynamic component, as in the corresponding simulations with a nonlinear Clausius-Clapeyron relationship. In the simulations with a linearized Clausius-Clapeyron relationship, the spatial scale of the precipitation changes is smaller, and the residual term in the water vapor budget decomposition is larger. The residual term is dominated by the component of the water vapor budget associated with changes in relative humidity $\delta \mathcal{H} \bar{q}_{s}$. That the magnitude of the thermodynamic term is smaller in the linearized Clausius-Clapeyron simulations is consistent with the smaller rate of change of the saturation vapor pressure with temperature: $\partial_{T} e_{s}^{\operatorname{lin}}<\partial_{T} e_{S}$ for $T>T_{0}$. This is partially offset by larger temperature changes in the simulations with a linearized Clausius-Clapeyron relationship.

The qualitative similarity of the response of tropical precipitation to orbital precession in simulations with a linearized Clausius-Clapeyron relationship is a more stringent test of the relative importance of the ClausiusClapeyron nonlinearity than the diagnostic approach of the previous section. The results support the conclusion that the Clausius-Clapeyron nonlinearity is not critical.

\section{e. Effects of surface heat capacity contrasts}

The simulations with an inhomogeneous surface heat capacity, which represents one aspect of the land-sea 
contrast in surface properties, have annual-mean precipitation changes that are qualitatively similar to the aquaplanet simulations: when perihelion occurs at the northern summer solstice, there is generally more annualmean precipitation in the Northern Hemisphere and less in the Southern Hemisphere compared with simulations having perihelion at the southern summer solstice (Fig. $3 d)$. However, the annual-mean precipitation changes are less antisymmetric about the equator, and the magnitudes of the dynamic and thermodynamic components of the precipitation change are comparable to each other.

The thermodynamic component of the changes in the water vapor flux convergence is still hemispherically antisymmetric, as in the aquaplanet case (Figs. 3a,d). The magnitude of the thermodynamic component is somewhat reduced compared with the aquaplanet case because the temperature change is smaller for the same insolation change as a result of the larger average surface heat capacity. Also, the spatial scale is somewhat different, a consequence of differences in the location and seasonal cycle of the convergence zones in the control climate, which is a dependence that we find across a range of orbital configurations (Merlis 2012).

The dynamic component of the precipitation changes has a more complicated structure than in the aquaplanet case. It is not hemispherically antisymmetric but has positive values over the southern part of the continent (near $10^{\circ} \mathrm{N}$ ), negative values equatorward of this, and positive values again in the Southern Hemisphere. The structure in the Northern Hemisphere arises because the intertropical convergence zone shifts northward during the summer. The convergence zone is collocated with the maximum in near-surface moist static energy or moist entropy (Privé and Plumb 2007a), which shifts northward in the bright summer because the response to increased insolation is more rapidly communicated to the atmosphere over the low surface heat capacity region than over the high surface heat capacity region, where there is more surface energy storage (Merlis et al. 2013b). The positive dynamic component of the precipitation change near $\sim 20^{\circ} \mathrm{S}$ is sufficiently large that it more than offsets the negative thermodynamic component, which leads to a region with more net precipitation during the dim summer. This shows that annual-mean precipitation does not always decrease in the hemisphere with the dim summer because, as in the aquaplanet simulations, the dynamic component of the precipitation changes can behave counterintuitively [see Schneider et al. (2012) for another example]. But the Clausius-Clapeyron nonlinearity remains unimportant in the dynamic precipitation changes: solely considering the annual-mean circulation change and the annualmean unperturbed specific humidity captures the dynamic precipitation changes (Merlis 2012). In other words, the annual-mean dynamic component of the precipitation change is proportional to the annualmean circulation changes (described in Merlis et al. 2013b); the seasonal cycle in specific humidity is not important.

In their analyses, Clement et al. (2004) and Hsu et al. (2010) found the dynamic component of the precipitation change to be large; however, this decomposition was only performed for solstice seasons. It is unclear what their solstice results imply for the annualmean precipitation, which is our focus here. Winter and summer precipitation changes may cancel in the annual mean because the precession forcing changes sign seasonally. Indeed, Fig. 3 of Hsu et al. (2010) shows substantial seasonal cancellation in their simulations. On the other hand, if circulation changes are limited to one season, the dynamic component of that season's precipitation change can be illuminating for the annual mean. In our simulations with surface heat capacity contrasts, circulation changes are largest in the summer solstice season (Merlis et al. 2013b), but this is not the case for the aquaplanet simulations (Merlis et al. 2013a). Therefore, one needs to understand how the factors that determine the atmospheric circulation's sensitivity to radiative perturbations vary seasonally, which we did in Merlis et al. (2013a) and Merlis et al. (2013b). In both sets of simulations, the dynamical regime of the circulations that gives rise to annual-mean changes is that of the angular momentum-conserving limit; the circulations are less sensitive to precession-forced insolation changes during the times of the year when they are influenced by the angular momentum fluxes of extratropical eddies (Merlis et al. 2013a,b).

We have also performed simulations with a simple representation of surface hydrology (Merlis et al. 2013b). The precipitation changes found in those simulations cannot be understood through the water vapor budget decomposition because, unlike the Asian monsoon regions, the continent region is in a desert regime where $P=E$, so changes in precipitation are met by changes in evaporation rather than by changes in the water vapor flux divergence. While this regime is of interest for African climate changes in the past (e.g., Su and Neelin 2005) and future (Held et al. 2005), it has complicated interactions between the surface and atmosphere (e.g., Lintner and Neelin 2009). These are not fully understood, even when potentially important vegetation feedbacks (e.g., Braconnot et al. 2007) are neglected. 


\section{Discussion and conclusions}

We have presented and analyzed the mechanisms that determine the tropical precipitation response to orbital precession in idealized GCM simulations. Consistent with proxy evidence, the annual-mean precipitation is greater in the hemisphere in which perihelion occurs in summer. This happens through a thermodynamic mechanism that acts even when the Clausius-Clapeyron nonlinearity is suppressed: specific humidity is increased seasonally where perihelion occurs in summer, leading to increased water vapor flux convergence and precipitation in the annual mean even if winds are unchanged, as illustrated in Fig. 1 (bottom). The relevant nonlinearity is an advective nonlinearity: the seasonal cycle of the precession-induced humidity changes is correlated with the seasonal cycle of the low-level convergence. This mechanism acts in regions in which precipitation occurs as the result of seasonal low-level convergence, so it is relevant in subtropical monsoon regions principally.

The thermodynamic mechanism can generate hemispherically antisymmetric subtropical precipitation changes on the time scales of orbital precession. When perihelion coincides with a hemisphere's summer, the lower atmosphere in that hemisphere is warmer and moister. Where summer is a season with climatological convergence and ascending motion (e.g., in subtropical monsoon regions), the additional water vapor alone leads to additional precipitation. In the opposite hemisphere, the summer season will have less insolation and a drier lower atmosphere, so there is less precipitation for the same magnitude of ascent. Our emphasis on thermodynamic changes (Fig. 1, bottom) is distinct from existing discussions of the precipitation response to orbital precession that have typically focused on changes in the strength of the atmospheric circulation (e.g., Ruddiman 2008), often conceptualized as seasonal sea-breeze circulations (Fig. 1, top).

While the thermodynamic mechanism can give rise to large-scale hemispherically antisymmetric precipitation changes even in the absence of atmospheric circulation changes, an alternative possibility is that dynamic changes are important for interpreting the speleothem record, as it is terrestrial. We have shown in Merlis et al. (2013b) that the atmospheric circulation at the continent boundary does, as expected, strengthen in summers with stronger insolation but that the surface inhomogeneity affects the atmospheric circulation through its effect on the atmospheric energy balance, not through the surface temperature, as the conventional view of monsoon circulations as seasonal land-sea breezes posits. The dynamic precipitation changes are from the atmospheric circulation's different sensitivity to radiative flux changes in winter and summer, which generates annualmean circulation changes (Merlis et al. 2013b). Analyzing proxies that are more sensitive to wind changes (e.g., upwelling) together with those that are more sensitive to precipitation changes (e.g., lake levels) may distinguish the relative importance of dynamic and thermodynamic mechanisms of precipitation change. For example, one might speculate that part of the difference in phasing of the Chinese speleothems and Arabian Sea upwelling proxies relative to the maximum precession insolation forcing (Clemens et al. 2010) arises from comparing a proxy that is sensitive to aspects of the hydrological cycle with a proxy that is sensitive to the surface wind. However, comparisons with proxy records also require consideration of the intermediate values of the longitude of perihelion that occur during the precession cycle and lower orbital eccentricity, which we do not address here [see Merlis (2012) for the precipitation changes in aquaplanet simulations with a wider range of orbital configurations]. Also, our emphasis here is on zonal and hemispheric mean changes, upon which regional variations with potentially different mechanisms and sensitivities will be superimposed, such as the eastwest variations found across South America by Cruz et al. (2009).

The speleothem record also contains hemispherically antisymmetric changes on time scales shorter than the $\sim 20$-kyr time scale of orbital precession. These "millennial events" are often interpreted as shifts in convergence zone location (e.g., Wang et al. 2007). In contrast, the thermodynamic mechanism for annual-mean precipitation changes in response to orbital precession does not depend on a displacement of circulation patterns. In fact, the convergence zones in the aquaplanet simulations do not shift substantially in latitude in response to orbital precession, nor does the fraction of the year that the convergence zone is in each hemisphere change (Fig. 6 of Merlis et al. 2013a). It is possible that the mechanism responsible for generating hemispherically antisymmetric changes is different for orbital precession than for shorter time scales, so care should be taken in the interpretation of records in which both time scales are present (e.g., Wang et al. 2006).

We have provided a new physical mechanism for annual-mean tropical (net) precipitation changes in response to orbital precession, motivated in part by the oxygen isotope record preserved in speleothems. The hemispheric-scale changes in annual-mean precipitation in our simulations are on the order of $1 \mathrm{~mm} \mathrm{day}^{-1}$, which may be exaggerated by aspects of the model formulation (e.g., the low surface thermal inertia and the large orbital eccentricity). The question remains how 
climate proxies such as speleothem oxygen isotopes respond to precession-induced changes in precipitation and other climate variables. To elucidate this question, mechanistic analyses of isotope-enabled GCM simulations of orbital precession would be helpful.

Acknowledgments. We appreciate comments by Andy Thompson, Jess Adkins, and three anonymous reviewers. This work was supported by a National Science Foundation Graduate Research Fellowship, a Princeton Center for Theoretical Science Fellowship, and National Science Foundation Grant AGS-1049201. The program code for the simulations, based on the Flexible Modeling System of the Geophysical Fluid Dynamics Laboratory, as well as the simulation results themselves, are available from the authors upon request.

\section{REFERENCES}

Anderson, J. L., and Coauthors, 2004: The new GFDL global atmosphere and land model AM2-LM2: Evaluation with prescribed SST simulations. J. Climate, 17, 4641-4673.

Ashkenazy, Y., I. Eisenman, H. Gildor, and E. Tziperman, 2010: The effect of Milankovitch variations in insolation on equatorial seasonality. J. Climate, 23, 6133-6142.

Boos, W. R., and Z. Kuang, 2010: Dominant control of the South Asian monsoon by orographic insulation versus plateau heating. Nature, 463, 218-222.

Bordoni, S., 2007: On the role of eddies in monsoonal circulations: Observations and theory. Ph.D. dissertation University of California, $195 \mathrm{pp}$.

—_, and T. Schneider, 2008: Monsoons as eddy-mediated regime transitions of the tropical overturning circulation. Nat. Geosci., 1, 515-519.

Braconnot, P., and Coauthors, 2007: Results of PMIP2 coupled simulations of the Mid-Holocene and Last Glacial MaximumPart 1: Experiments and large-scale features. Climate Past, 3, 261-277.

Brayshaw, D. J., B. Hoskins, and E. Black, 2010: Some physical drivers of changes in the winter storm tracks over the North Atlantic and Mediterranean during the Holocene. Philos. Trans. Roy. Soc., 368A, 5185-5223.

Clemens, S. C., W. L. Prell, and Y. Sun, 2010: Orbital-scale timing and mechanisms driving Late Pleistocene Indo-Asian summer monsoons: Reinterpreting cave speleothem $\delta^{18} \mathrm{O}$. Paleoceanography, 25, PA4207, doi:10.1029/2010PA001926.

Clement, A. C., A. Hall, and A. J. Broccoli, 2004: The importance of precessional signals in the tropical climate. Climate Dyn., 22, 327-341.

COHMAP members, 1988: Climatic changes of the last 18,000 years: Observations and model simulations. Science, 241, 1043-1052.

Cruz, F. W., and Coauthors, 2005: Insolation-driven changes in atmospheric circulation over the past 116,000 years in subtropical Brazil. Nature, 434, 63-66.

— of South American precipitation. Nat. Geosci., 2, 210-214.

Dayem, K., D. S. Battisti, G. H. Roe, and P. Molnar, 2010: Lessons learned from the modern monsoon applied to the interpretation of paleoclimate records. Earth Planet. Sci. Lett., 295, 219-230.

deMenocal, P., J. Ortiz, T. Guilderson, J. Adkins, M. Sarnthein, L. Baker, and M. Yarusinsky, 2000: Abrupt onset and termination of the African Humid Period: Rapid climate responses to gradual insolation forcing. Quat. Sci. Rev., 19, 347-361.

Frierson, D. M. W., 2007: The dynamics of idealized convection schemes and their effect on the zonally averaged tropical circulation. J. Atmos. Sci., 64, 1959-1976.

Gasse, F., and Coauthors, 1991: A 13,000-year climate record from western Tibet. Nature, 353, 742-745.

Held, I. M., 2000: The general circulation of the atmosphere. Fellows Project Rep. WHOI-2001-03, Woods Hole Oceanographic Institution, Woods Hole, MA, 179 pp. [Available online at https://darchive.mblwhoilibrary.org/handle/1912/15.]

_ 2001: The partitioning of the poleward energy transport between the tropical ocean and atmosphere. J. Atmos. Sci., 58, 943-948.

— tions in a nearly inviscid atmosphere. J. Atmos. Sci., 37, 515533.

— cycle to global warming. J. Climate, 19, 5686-5699.

— T. L. Delworth, J. Lu, K. L. Findell, and T. R. Knutson, 2005: Simulation of Sahel drought in the 20th and 21st centuries. Proc. Natl. Acad. Sci. USA, 102, 17 891-17 896.

Herzschuh, U., 2006: Palaeo-moisture evolution in monsoonal central Asia during the last 50,000 years. Quat. Sci. Rev., 25, 163-178.

Hewitt, C. D., and J. F. B. Mitchell, 1998: A fully coupled GCM simulation of the climate of the mid-Holocene. Geophys. Res. Lett., 25, 361-364.

Hsu, Y.-H., C. Chou, and K.-Y. Wei, 2010: Land-ocean asymmetry of tropical precipitation changes in the mid-Holocene. J. Climate, 23, 4133-4151.

Huybers, P., and C. Wunsch, 2003: Rectification and precession signals in the climate system. Geophys. Res. Lett., 30, 2011, doi:10.1029/2003GL017875.

Jackson, C. S., and A. J. Broccoli, 2003: Orbital forcing of Arctic climate: Mechanisms of climate response and implications for continental glaciation. Climate Dyn., 21, 539-557.

Joussaume, S., and Coauthors, 1999: Monsoon changes for 6000 years ago: Results of 18 simulations from the Paleoclimate Modeling Intercomparison Project (PMIP). Geophys. Res. Lett., 26, 859-862.

Khon, V. C., W. Park, M. Latif, I. I. Mokhov, and B. Schneider, 2010: Response of the hydrological cycle to orbital and greenhouse gas forcing. Geophys. Res. Lett., 37, L19705, doi:10.1029/2010GL044377.

Kutzbach, J. E., 1981: Monsoon climate of the early Holocene: Climate experiment with the Earth's orbital parameters for 9000 years ago. Science, 214, 59-61.

_ parameters and surface boundary conditions on climate simulations for the past 18000 years. J. Atmos. Sci., 43, 1726-1759.

Legrande, A. N., and G. A. Schmidt, 2009: Sources of Holocene variability of oxygen isotopes in paleoclimate archives. Climate Past, 5, 441-455.

Lindzen, R. S., and A. Y. Hou, 1988: Hadley circulations for zonally averaged heating centered off the equator. J. Atmos. Sci., 45, 2416-2427.

Lintner, B. R., and J. D. Neelin, 2009: Soil moisture impacts on convective margins. J. Hydrometeor., 10, 1026-1039. 
Merlis, T. M., 2012: The general circulation of the tropical atmosphere and climate changes. Ph.D. dissertation California Institute of Technology, 185 pp. [Available online at http:// resolver.caltech.edu/CaltechTHESIS:07012011-191902511.]

_ , T. Schneider, S. Bordoni, and I. Eisenman, 2013a: Hadley circulation response to orbital precession. Part I: Aquaplanets. J. Climate, 26, 740-753.

,,--- , and,$- 2013 \mathrm{~b}$ : Hadley circulation response to orbital precession. Part II: Subtropical continent. J. Climate, 26, 754-771.

O'Gorman, P. A., and T. Schneider, 2008: The hydrological cycle over a wide range of climates simulated with an idealized GCM. J. Climate, 21, 3815-3832.

Pausata, F. S. R., D. S. Battisti, K. H. Nisancioglu, and C. M. Bitz, 2011: Chinese stalagmite $\delta^{18} \mathrm{O}$ controlled by changes in the Indian monsoon during a simulated Heinrich event. Nat. Geosci., 4, 474-480.

Prell, W. L., and J. E. Kutzbach, 1987: Monsoon variability over the past 150,000 years. J. Geophys. Res., 92 (D7), 8411-8425.

Privé, N. C., and R. A. Plumb, 2007a: Monsoon dynamics with interactive forcing. Part I: Axisymmetric studies. J. Atmos. Sci., 64, 1417-1430.

_ and 2007b: Monsoon dynamics with interactive forcing. Part II: Impact of eddies and asymmetric geometries. $J$. Atmos. Sci., 64, 1431-1442.

Ruddiman, W. F., 2008: Earth's Climate: Past and Future. 2nd ed. W. H. Freeman and Company, 388 pp.

Schmidt, G. A., D. T. Shindell, and S. Harder, 2004: A note on the relationship between ice core methane concentrations and insolation. Geophys. Res. Lett., 31, L23206, doi:10.1029/ 2004GL021083.
Schneider, T., 2006: The general circulation of the atmosphere. Annu. Rev. Earth Planet. Sci., 34, 655-688.

, P. A. O'Gorman, and X. J. Levine, 2010: Water vapor and the dynamics of climate changes. Rev. Geophys., 48, RG3001, doi:10.1029/2009RG000302.

, S. D. B. Graves, E. L. Schaller, and M. E. Brown, 2012: Polar methane accumulation and rainstorms on Titan from simulations of the methane cycle. Nature, 481, 58-61.

Seager, R., N. Naik, and G. A. Vecchi, 2010: Thermodynamic and dynamic mechanisms for large-scale changes in the hydrological cycle in response to global warming. J. Climate, 23, 4651-4668.

Street, F. A., and A. T. Grove, 1979: Global maps of lake-level fluctuations since 30,000 yr BP. Quat. Res., 12, 83-118.

Su, H., and J. D. Neelin, 2005: Dynamical mechanisms for African monsoon changes during the mid-Holocene. J. Geophys. Res., 110, D19105, doi:10.1029/2005JD005806.

Wang, X., A. S. Auler, R. L. Edwards, H. Cheng, E. Ito, and M. Solheid, 2006: Interhemispheric anti-phasing of rainfall during the last glacial period. Quat. Sci. Rev., 25, 33913403 .

Y. Wang, X. Kong, and M. Solheid, 2007: Millennial-scale precipitation changes in southern Brazil over the past 90,000 years. Geophys. Res. Lett., 34, L23701, doi:10.1029/2007GL031149.

Wang, Y., and Coauthors, 2008: Millennial- and orbital-scale changes in the East Asian monsoon over the past 224,000 years. Nature, 451, 1090-1093.

Zhao, Y., and Coauthors, 2005: A multi-model analysis of the role of the ocean on the African and Indian monsoon during the mid-Holocene. Climate Dyn., 25, 777-800. 